\title{
Progress on Modeling of Dynamic Productivity of Fractured Gas Condensate Reservoir Based on a Fluid-Solid Coupling Method
}

\author{
Shuai Wang *, Xianhong Tan, Yang Xia, Bo Tian and Bin Liang \\ China National Offshore Oil Corporation Research Institute, Beijing, China
}

Bozhong 19-6 gas field is the first discovered large-scale gas condensate field in eastern China, which is also one of the largest metamorphic rock gas condensate fields in the world. It is a buried hill type, low permeability reservoir, with ultra-high condensate content where the fluid is nearly at its dew point pressure. No similar experience with such reservoirs have previously been reported in the context of gas field development in China

OPEN ACCESS

Edited by:

Yuwei Li,

Liaoning University, China

Reviewed by:

Zhao Hailong,

China University of Petroleum

(Huadong), China

Qianru Qi,

University of Southern California,

United States

Daiyin Yin,

Northeast Petroleum University, China

*Correspondence:

Shuai Wang

408207747@qq.com

$$
\begin{array}{r}
\text { Specialty section: } \\
\text { This article was submitted to } \\
\text { Economic Geology, } \\
\text { a section of the journal } \\
\text { Frontiers in Earth Science }
\end{array}
$$

Received: 09 June 2021 Accepted: 11 October 2021 Published: 25 November 2021

Citation:

Wang S, Tan X, Xia Y, Tian B and Liang B (2021) Progress on Modeling of Dynamic Productivity of Fractured Gas Condensate Reservoir Based on a

Fluid-Solid Coupling Method.

Front. Earth Sci. 9:722787.

doi: 10.3389/feart.2021.722787 and step-by-step progresses is been made to characterize this reservoir. Overall, documentation concerning this type of reservoir is rarely seen worldwide. This paper includes key successful results from multiple perspectives including experiments correlations, numerical modeling and the significance of incorporating certain details. Based on a fluid-solid coupling method, the simulations consider several factors including the fracture distribution, low permeability, medium deformation, and condensate characteristics, as well as their effects on the gas productivity. In the laboratory experiments, the stress sensitivity of the rock was tested using representative core samples. Here, experiment-based correlations of the starting pressure gradient of the gas condensate reservoir are proposed. The starting pressure gradient of different fluid types, such as black oil and gas condensate are highlighted as accurately simulating the reservoir. As a result, the numerical model to predict the dynamic productivity of a single well was successfully established considering all those factors. This paper can serve as a reference for studying other studies of metamorphic, fractured gas condensate reservoirs.

Keywords: fractured gas condensate reservoir, dynamic productivity of gas wells, fluid-solid coupling, numerical simulation, prediction

\section{INTRODUCTION}

Fractured gas reservoirs are widely distributed in China (Jiang and Wang, 2009; Huang et al., 2021), such as Ke Shen-8 gas reservoir in Tarim Basin and Mo Xijia-2 gas reservoir in Sichuan Basin. The Bozhong 19-6 gas condensate field is the first discovered buried hill, large condensate field with low permeability, extremely high condensate content in eastern China, which fluid is nearly at its dew point pressure. It is also one of the largest condensate fields of metamorphic rock in the world. The geological reserve has a volume of nearly 1 billion cubic meters. While it is challenging to develop the gas field, there was no similar experience domestically for reference purposes. In the development of this type of condensate gas reservoir, condensate oil will be precipitated after the local formation pressure is lower than the dew point pressure. In addition, the reservoir has low permeability; the 


\section{A}

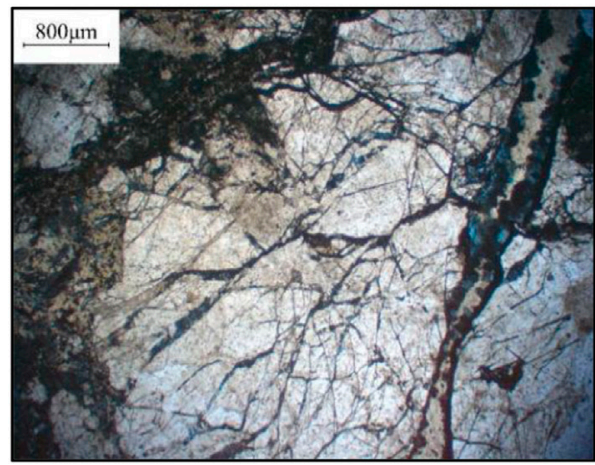

in casting thin sections
B

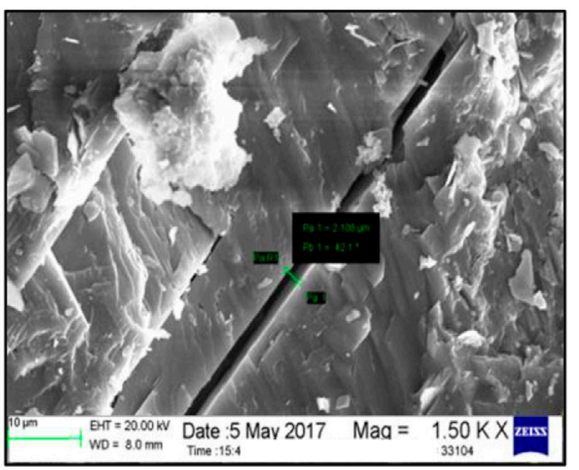

under scanning electron microscope

FIGURE 1 | Demonstrations of microfracture distribution. (A) In casting a thin sections, (B) Under a scanning electron microscope.

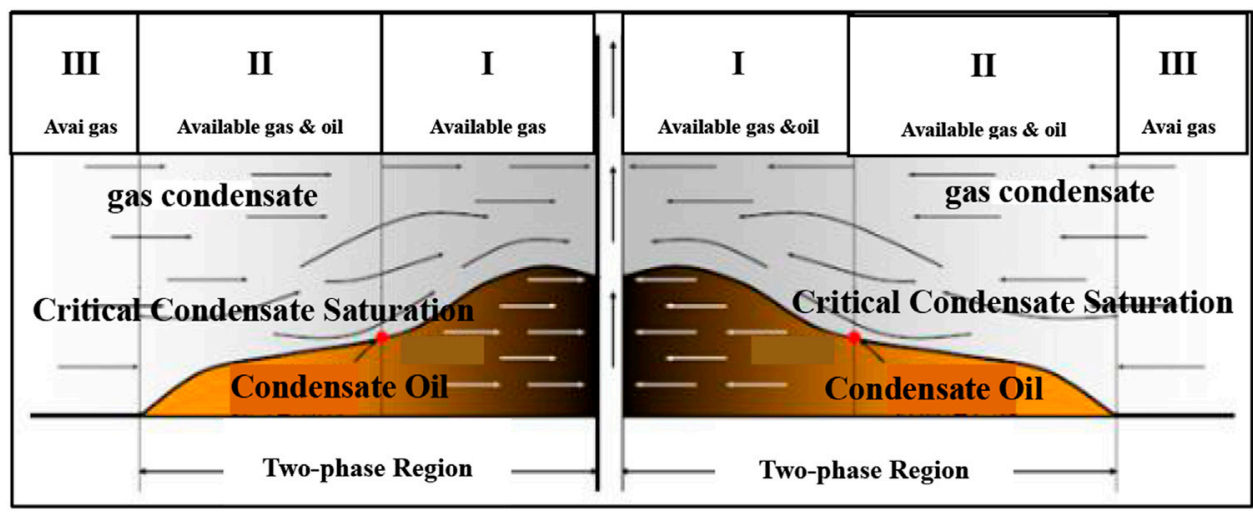

FIGURE 2 | Distribution of condensate near the wellbore zone.

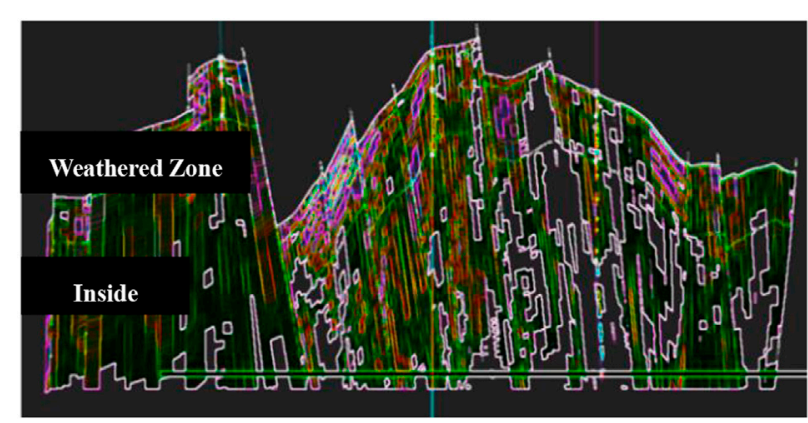

FIGURE 3 | Geological model of fractured reservoir in buried hill. Experiments on testing stress sensitivity of the fracture-matrix system.

matrix permeability is only $0.3 \mathrm{mD}$. Both condensate oil and gas are difficult to be recover after precipitation, resulting in low single well productivity and recovery. Accurate prediction of production capacity of this specific type of reservoir are needed, because such predictions form foundation for planning the gas field development to achieve the optimized recovery.

A lot of research work has been done on fractured reservoirs. In 1963, Warren J. E. took the established theoretical model for natural fractured reservoirs, which was the first one to study the characteristics of fracture-matrix structure (Warren and Root, 1963). In 1987, Ozkan E. proposed a way to predict the productivity from a single well in a natural fractured reservoir, under the constant pressure boundary condition, using the real production data. The model defined five different flow mechanisms and preliminarily proposed a method for estimating the reservoir parameters (Ozkan et al., 1987). At present, the equivalent continuum theory is mostly adopted in calculating the productivity of fractured gas reservoirs ( $\mathrm{Li}$ et al., 2016). The method simplifies the fracture distribution within the reservoir, but does not consider the geometry and location of fractures. Assuming the steady state for gas condensate reservoir, Guo et al. proposed an equation to predict the productivity for the case of gas-liquid multiphase 
TABLE 1 | Core parameters of different permeability and fracture distribution.

\begin{tabular}{|c|c|c|c|c|c|}
\hline Core no & Length $(\mathrm{cm})$ & Diameter (cm) & Porosity (\%) & Permeability (mD) & $\begin{array}{l}\text { Existence of } \\
\text { fracture }\end{array}$ \\
\hline 1 & 10.30 & 6.57 & 9.76 & 3.38 & Fractured \\
\hline 2 & 9.25 & 9.36 & 7.54 & 2.95 & Fractured \\
\hline 4 & 11.28 & 6.59 & 8.14 & 0.71 & Crack-free \\
\hline 5 & 8.30 & 6.00 & 3.66 & 0.07 & Crack-free \\
\hline 6 & 10.85 & 6.00 & 8.20 & 0.71 & Crack-free \\
\hline
\end{tabular}

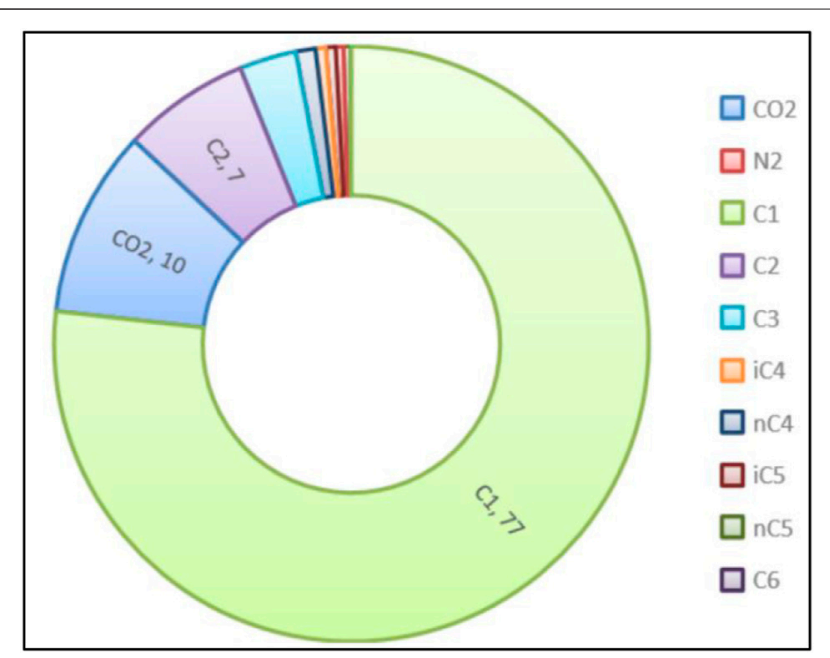

FIGURE 4 | Composition distribution of flash gas sampled from BZ19-6$X$ gas condensate well.

flow, where the open flow rate of gas well can be calculated by software (Guo, 2007). Li established a three-dimensional nonDarcy flow model for the system of oil and water. However, because of its high computational demand, this method has not been widely adopted.

In this paper, a numerical model is developed based on previous progress, incorporating specifically stress sensitivity and starting pressure gradient distributions (Lu et al., 2018). The model uses the fluid-solid coupling method and considers several factors, including the fracture distribution, medium variation in the low permeability, fractured gas reservoir, and reverse condensate volume. The incorporation of the starting pressure gradient of the gas condensate reservoir is highlighted here, and the relationship between pressure and permeability is correlated with experimental data.

\section{METHODOLOGY}

\section{The Productivity of Fractured Gas Reservoirs}

Buried hill gas condensate reservoir is a very complex type of reservoir with higher productivity than that of low permeability sandstone gas condensate reservoir. Eq. 1 is the conventional

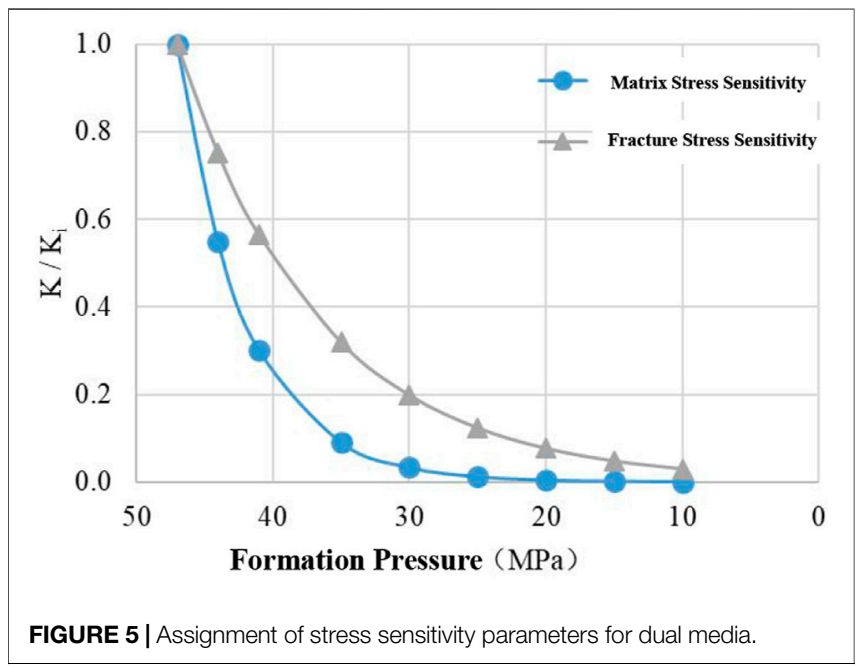

method to calculate gas well productivity (Sun et al., 2012). The gas well production capacity is affected by not only rock and fluid properties but also well condition such as skin factor of a well.

$$
q_{g}=\frac{0.07852 K h\left(p_{R}^{2}-p_{w f}^{2}\right)}{\mu_{\mathrm{g}} Z T\left(\ln \frac{r_{e}}{r_{w}}+S_{a}\right)},
$$

where $q_{\mathrm{g}}$ is gas production rate $\left(\mathrm{m}^{3} /\right.$ day), $K$ is the permeability $(\mathrm{mD}), p_{\mathrm{R}}$ is reservoir pressure $(\mathrm{MPa}), p_{w f}$ is bottom hole flowing pressure (MPa), $Z$ is gas compression factor, $T$ is temperature of reservoir $\left({ }^{\circ} \mathrm{C}\right)$, and $S_{a}$ is skin factor of the well.

\section{Diagenesis of the Reservoir Rock}

The Bozhong 19-6 reservoir is deeply bedded and highly heterogeneous, as which has experienced several tectonic movement periods and stress mode changes. Figure 1 shows the morphology of micro-fractures from a thin section of the rock sample from the reservoir. Influenced by its depth and high compaction, generally the openings of macro-fractures are small. From imaging logging, those fracture openings are within $100 \sim 400 \mu \mathrm{m}$. From the thin section, the openings of microfractures are about $20 \sim 30 \mu \mathrm{m}$. Fractures cut through each other, and their distributions have multi-stage fracture characteristics. It is concluded that the fracture distribution is the most important factor controlling the physical properties of 


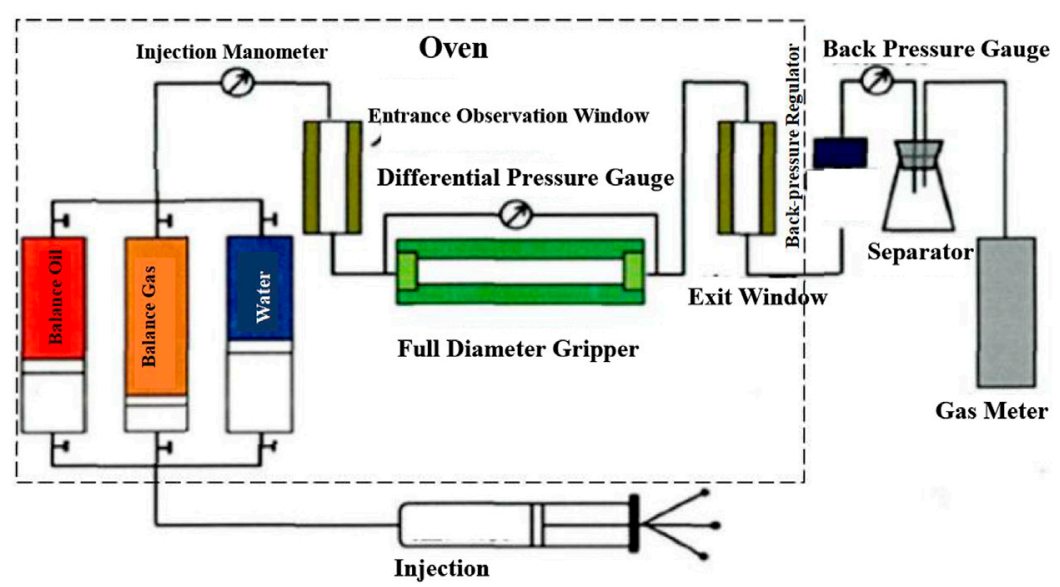

FIGURE 6 | Illustration of equipment measuring starting pressure gradient of the gas condensate reservoir (Chen et al., 2019).

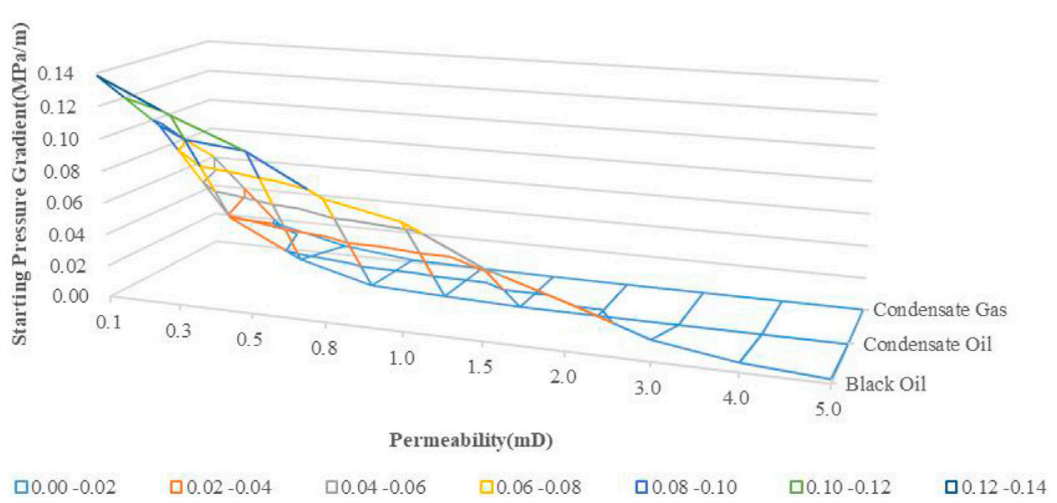

FIGURE 7 | Starting pressure gradient with respect to permeability and different fluid types (Wang et al., 2017).

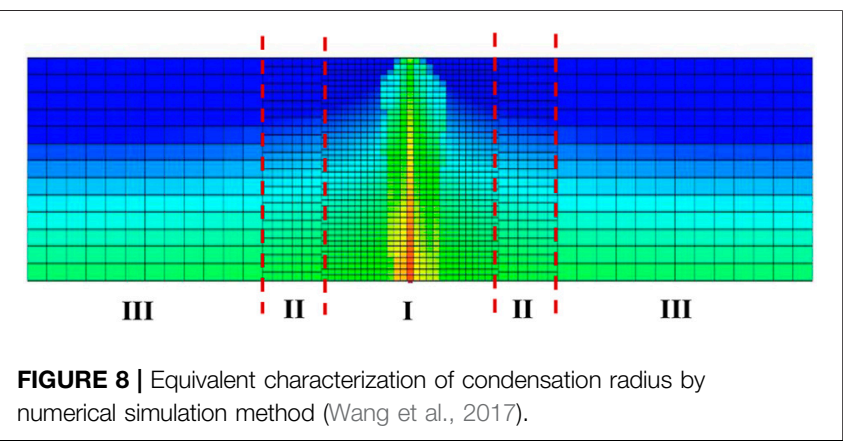

the reservoir, that is, the fractures contribute the most of fluid flow. In the matrix, the radii of pore throats are less than $1 \mu \mathrm{m}$. Small pores and their poor connectivity result in low permeability. Overall, the flow velocity of the fluid is very low and the flow is highly nonlinear.

The starting pressure gradient is closely related to the permeability of the porous media. Generally speaking, the lower the permeability corresponds to the higher the starting pressure gradient. In addition, the influence of pressure change on permeability of the fracture-matrix structure cannot be ignored. The formation pressure decline along the gas field development process changes the effective confining stresses. As the permeability decreases continuously, this process is irreversible. The aforementioned factors all lead to a decrease in productivity as the recovery continues. Therefore, the productivity is dynamic and must be modeled.

\section{Effect of Fluid Phase Changes}

The change of fluid phase state in gas condensate reservoir is a very important factor affecting the dynamic productivity. Compared with dry gas well, gas condensate well has a faster productivity decline. This is because when the bottom hole flowing pressure is lower than the dew point pressure, condensate forms in the area around the well, which leads to additional pressure losses, and negatively affects the movement of gas. When the critical saturation for condensate fluid is exceeded, the condensate begins to flow in the reservoir. Figure 2 shows the hydrocarbon two-phase flow in the nearwellbore zone. 
TABLE 2 | Gas condensate parameters of well BZ19-6-X (Liang et al., 2020).

\begin{tabular}{|c|c|c|c|c|c|c|c|c|}
\hline $\begin{array}{l}\text { Sampling } \\
\text { horizon }\end{array}$ & $\begin{array}{l}\text { GOR } \\
\mathrm{m}^{3} / \mathrm{m}^{3}\end{array}$ & $\begin{array}{c}\text { Dew point } \\
\text { pressure }<\mathrm{MPa}\end{array}$ & $\begin{array}{c}\text { Dew } \\
\text { pressure } \\
\text { difference } \\
\text { MPa }\end{array}$ & $\begin{array}{c}\text { Condensate } \\
\text { content } \\
\mathrm{g} / \mathrm{m}^{3}\end{array}$ & $\begin{array}{c}\text { Maximum } \\
\text { amount } \\
\text { of reverse } \\
\text { condensate } \\
\%\end{array}$ & $\begin{array}{c}\text { Surface } \\
\text { condensate } \\
\text { density } \\
\text { t/m }\end{array}$ & $\begin{array}{l}\text { Gaseous } \\
\text { Z-factor }\end{array}$ & $\begin{array}{c}\text { Gas volume } \\
\text { coefficient }\end{array}$ \\
\hline $\mathrm{Ar}$ & 1,095 & 45.61 & 1.9 & 710.67 & 40.97 & 0.798 & 1.217 & 0.00381 \\
\hline
\end{tabular}

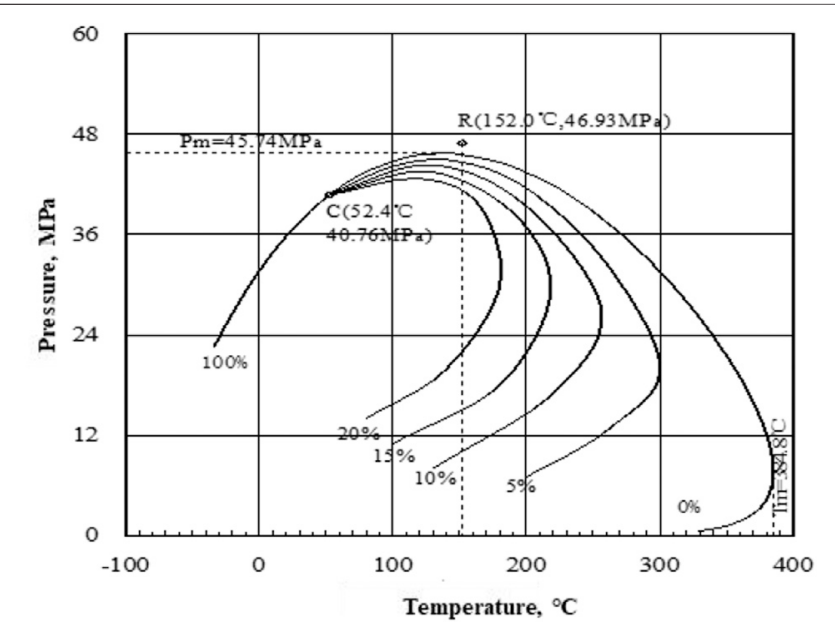

FIGURE 9 | Phase envelope of Bozhong 19-6 gas condensate field (Liang et al., 2020).

\section{Predictive Model of Dynamic Productivity}

Based on the Petrel integrated software platform, a threedimensional geological model of the fracture-matrix system was established, which adopts the technology of DFN stochastic modeling. Combined with the sensitive seismic attributes, the model considered various factors such as paleogeomorphology, fault and micro-geomorphology, to make a quantitative prediction of the fractured reservoir. Figure 3 shows the matrix-fracture coupling geological model of Bozhong 19-6 reservoir. It includes the dual geological framework and shows the layering of weathering zone in details.

1) Modeling of the matrix

Multiple parameters such as fracture density, net-gross ratio, matrix porosity, and permeability were calibrated by microseismic data. Those parameters are used to identify the characteristics of the matrix, according to the scheme in matrix classification. Through probabilistic simulation with constraints representing various types of matrices, distribution of different matrix types can be established.

\section{2) Modeling of fracture}

For the fractures of different scales, the combined deterministic simulation and probabilistic simulation were used to model the fracture network. For large fractures at fault level, the fracture distribution detected by seismic attributes such as maximum curvature and refined probabilistic fault volume were used for input, which can be directly transformed into the large-scale fracture network through deterministic simulation. For medium-sized fractures, fracture distribution interpreted with imaging logging was used.

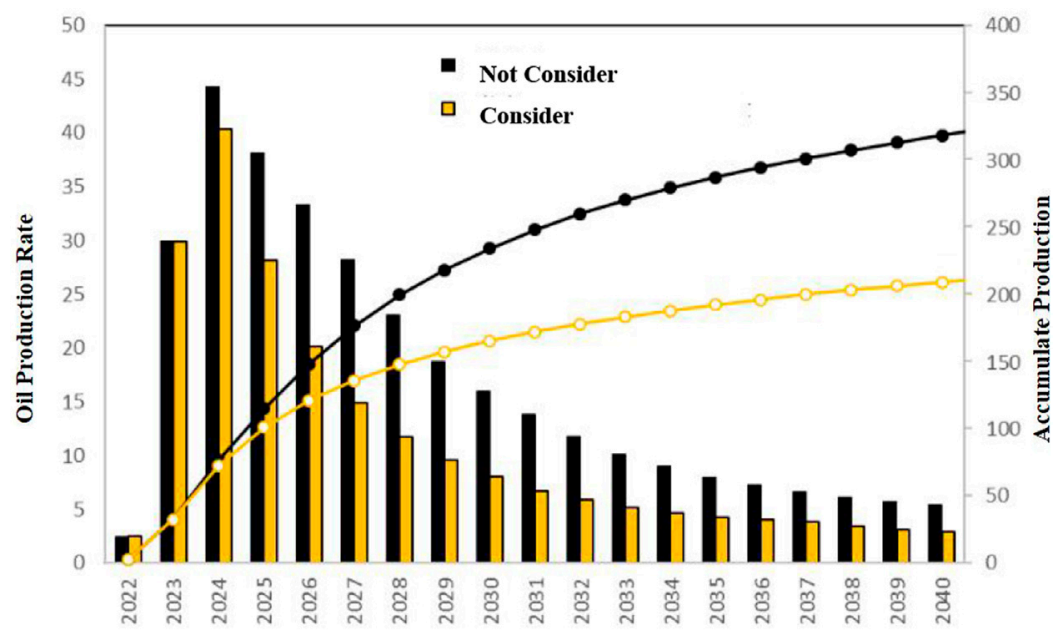

FIGURE 10 | Effect of stress sensitivity on oil production prediction. 


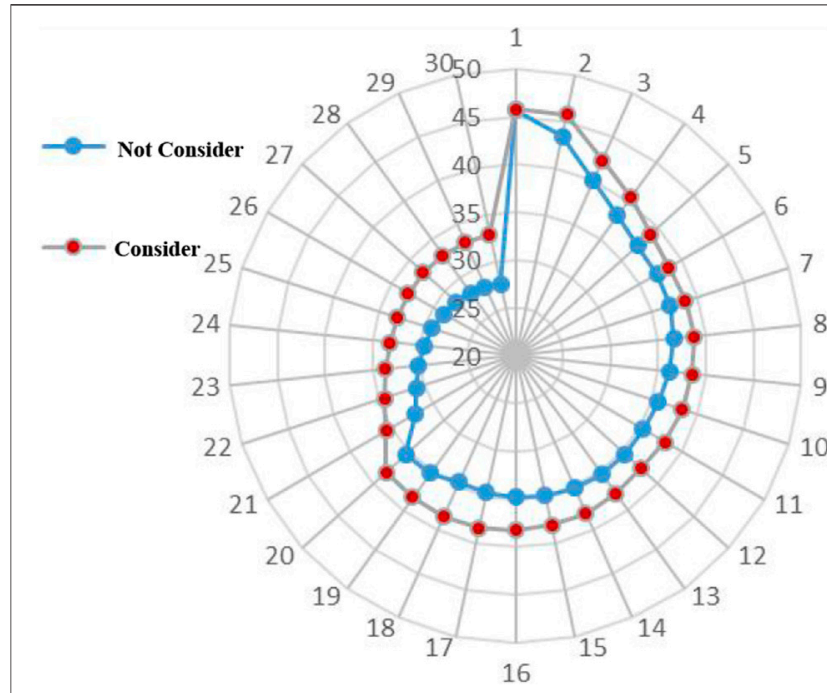

FIGURE 11 | Effect of stress sensitivity on bottom hole pressure.

\section{Experiments Testing the Stress Sensitivity}

As the numerical modeling requires a pressure-permeability curve, experiments on stress sensitivity were conducted, using cores of different fracture distributions and in situ fluid sample. Six core samples with different levels in permeability and fracture distribution were selected to conduct the experiments testing stress sensitivity, using high-temperature and high-pressure core displacement device. The fracture density was 4 per meter. Core parameters are shown in Table 1, in which cores 1 3 represent fractured rock and cores $4 \sim 6$ represent crack-free rock. The condensate fluid sampled from well BZ19-6-X, as shown in Figure 4, was used in the experiments.

The experimental procedure under unsteady-state condition is as follows:

1) Place the sample into core holder and maintain a constant pressure drop;

2) Change the confining pressure through a manual pump, and test the core permeability when the confining pressure reaches $10,20,30,40$, and $50 \mathrm{MPa}$, respectively;

3) Correlate the core permeability changes with respect to effective stress.

Based on the experimental results, deformation coefficient of the fractured rock was obtained, as shown in Eq. 2. Considering the stress-sensitive effect, the relationship between permeability and formation pressure is shown in Eq. 3. The pressure-permeability curves for both fracture and matrix are shown in Figure 5.

$$
\begin{aligned}
& \alpha_{K}=0.153 K_{i}^{-0.34}, \\
& K=K_{i} \mathrm{e}^{-\alpha_{K}\left(p_{i}-p\right)},
\end{aligned}
$$

where $\alpha_{k}$ is the medium deformation coefficient, $K$ is the permeability of reservoir $(m D), K_{i}$ is the initial permeability

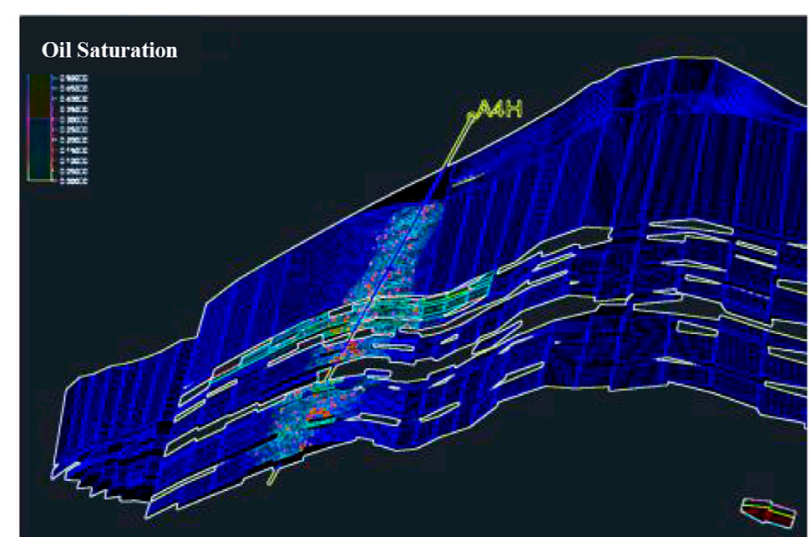

FIGURE 12 | Prediction result of condensate precipitation in well BZ196-X (Gao et al., 2020).

of reservoir $(m D), p$ is the reservoir pressure $(m D)$, and $p_{i}$ is the initial reservoir pressure $(m D)$.

\section{Starting Pressure Gradient}

The actual fluid flow in the formation is under high temperature and pressure. To better describe the non-Darcy flow, the starting pressure gradient of the gas condensate flow under high temperature and pressure was studied through experimental work (Chen et al., 2019). Under the pressure of $50 \mathrm{MPa}$ and temperature of $152^{\circ} \mathrm{C}$, the cores (with fractures) with permeability of 2.51 and $2.58 \mathrm{mD}$, respectively, were used in testing with different fluid samples. The experimental set was composed of an injection pump system, core gripper, back pressure regulator, differential pressure gauge, temperature control system, liquid fraction collector, and gas meter. The flow velocity of the gas condensate through the cores was controlled by changing the pressure drop rate at the outlet, using the back pressure regulator. Illustration of the experimental equipment is shown in Figure 6.

Characterization of starting pressure gradient in the gas condensate reservoir had also been done through numerical simulation (Wang et al., 2017). In the study, black oil and gas condensate were studied, as shown in Figure 7. Figure 8 shows the estimated distribution of liquid condensate, which honored the theoretical condensate distribution around the wellbore.

\section{RESULTS AND DISCUSSIONS}

Bozhong 19-6 gas condensate reservoir was characterized for both matrix and fracture system. The fracture analysis was conducted for various magnitudes, including macro-fractures, medium and small fractures, and micro-fractures (Kang et al., 2021). The total porosity of the reservoir rock in Bozhong 19-6 ranges from 2 to $4 \%$, and the permeability ranges from 0.3 to $4 \mathrm{mD}$, showing the characteristics of low porosity and low permeability. The Bozhong 19-6 gas condensate reservoir is characterized by high condensate content, and its maximum 


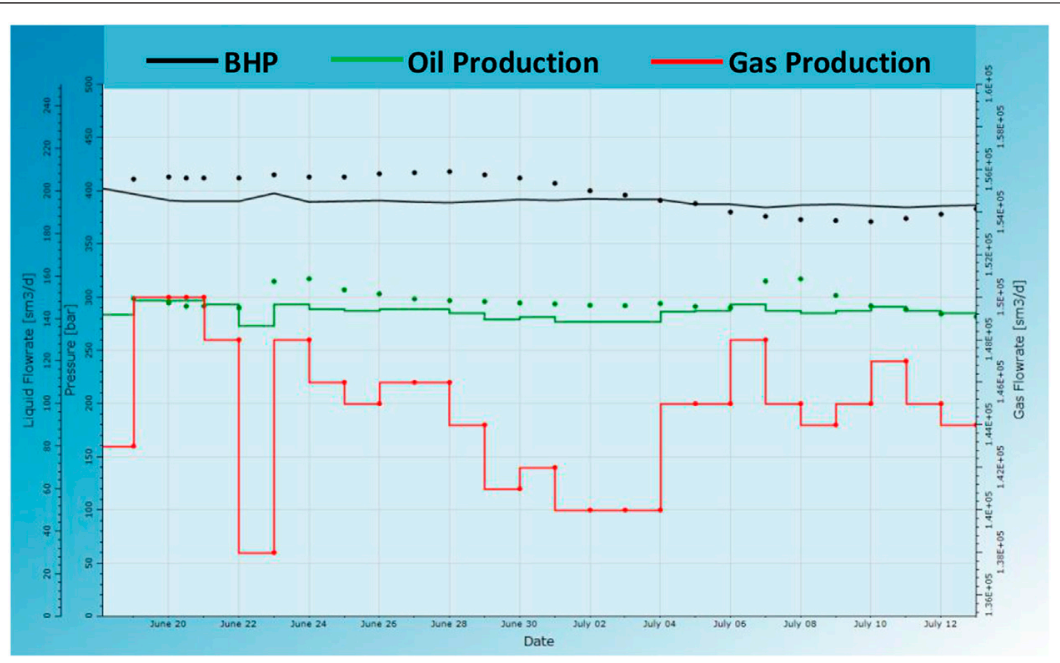

FIGURE 13 | Historical matching effect of well BZ19-6-X (Gao et al., 2020).

reversed condensate volume can be as high as $40.97 \%$, as shown in Table 2, and small ground-dew pressure difference of $1.9 \mathrm{MPa}$ (Liang et al., 2020). When the pressure drop increases, precipitated liquid will clog the near-wellbore area in the formation, resulting in reduced production, as shown in Figure 9.

Incorporating the stress sensitivity and starting pressure gradient mentioned previously in the numerical simulation, it was noticed that as the reservoir development continues, the influence of stress sensitivity and starting pressure gradient on the performance becomes more significant, as shown in Figure 10 and Figure 11. Their effects are mainly manifested as rapid production decline and increased loss in bottom hole flowing pressure. Compared with the case not including the stress sensitivity, the difference on estimated average pressure drop is $10 \%$; therefore, the difference on cumulative production estimation is much more. Comparing the cases that do and do not including the starting pressure gradient curves, the difference predicted oil production can as high as $72 \%$. Therefore, stress sensitivity and starting pressure gradient cannot be neglected in modeling the specific type of reservoir.

Based on the fluid-solid coupling model (Gao et al., 2020) established, the productivity of BZ19-6-X well was predicted, and historical fitting was performed. Figure 12 and Figure 13 show the history match and prediction of retrograde condensate production in Well BZ19-6-X. The well was managed by controlling gas production rate and pressure drawdown. Through fitting the condensate production data, the accuracy in prediction of the single well production history was more than $90 \%$.

\section{CONCLUSION}

1) Based on fluid-solid coupling numerical model and relevant experimental work, this paper comprehensively reviewed the key factors that must be incorporated into such a model, including fracture distribution, permeability changes for gas condensate reservoirs, condensate volume, non-Darcy flow, and other factors affecting well productivity.

2) A comparison with experimental data indicates that the starting pressure gradient of different fluid types of black oil and gas condensate, as well as the stress sensitivity, are key factors that should be included in numerical simulation of the fractured gas condensate reservoir.

3) According to validation with real data, the numerical model was able to predict the performance of a single well, with a fitting accuracy of more than $90 \%$.

In the review of the key progress made toward characterizing this rare type of reservoir from various perspectives and scales, the final developed model was able to predict the performance of a single well. Studies that have been done on the metamorphic, fractured gas condensate reservoir have resulted in significant progress. This paper may serve as a reference for other reserves of the same type.

\section{DATA AVAILABILITY STATEMENT}

The original contributions presented in the study are included in the article/Supplementary Material. Further inquiries can be directed to the corresponding author.

\section{AUTHOR CONTRIBUTIONS}

All authors listed have made a substantial, direct, and intellectual contribution to the work and approved it for publication.

\section{ACKNOWLEDGMENTS}

This paper is supported by CNOOC Research Institute. 


\section{REFERENCES}

Chen, J., Li, J., Zhou, Z., and Wang, Y. B. (2019). Establishment and Application of Production Capacity Formula of Wenchang Low Permeability Gas Condensate Well [J]. SHANDONG CHEMICAL INDUSTRY 48 (19).

Gao, Z. N., Geng, Z. G., Zhang, F. Y., Yu, Y. Z., and Wu, T. T. (2020). Numerical Simulation of Metamorphis Buried-Hill Fractured Gas Condensate Reservoir Based on Fluid-Sloid Coupling [J]. Spec. Oil Gas Reservoirs 02 (20). doi:10.3969/j.issn.1006-6535.2020.01.018

Guo, K. L., Guo, Q., and Cheng, S. Q. (2007). Study on Productivity Calculation Model and Method of Horizontal Well in Condensate Gas Reservoir [J]. Lithologic Reservoir 07 (01).

Huang, L., Kang, Y., and Xu, C. (2021). Comprehensive Evaluation of Gas Condensate Reservoir Productivity Based on Fracture Characteristic Parameters [J]. Petrochemical Appl. 21 (03).doi:10.1016/j.jngse.2014.03.016

Jiang, W. K., and Wang, J. M. (2009). Productivity Evaluation of Gas Well in Fractured Carbonate Reservoir [J]. Nat. Gas Industry 29 (9), 103-105. doi:10.3787/j.issn.1000-0976.2009.09.029

Kang, B. T., Zhang, Y. C., Chen, G. N., Li, C. X., Jiang, B., and Zuo, J. Y. (2021). A New Development Index Prediction Method for Confined Gas Condensate Reservoirs [J]. Petrochemical Appl. (01), 21. doi:10.3969/j.issn.1673-5285.2021.01.007

Li, Q., Guo, Y., Tian, C., Qian, L., and Cheng, Y. (2016). Different Angle Crack Defects on the Influence of Dynamic Fracture Behavior of Materials [J]. Sci. Tech. Eng. 18 (28), 1-5.

Liang, B., Tan, X. H., Zhu, G. J., Tian, B., Wang, S., Li, N., et al. (2021). Study on Improving Recovery of Condensate Oil in Low Perm and High Condensate Gas Reservoir. Singapore: Springer. doi:10.1007/978-981-16-0761-5_293

Lu, J. L., Zhang, H., Chang, B. H., Cao, W., and Sun, H. D. (2018). A New Method for Productivity Evaluation of Gas Condensate Well in Gas-Liquid Two-phase State [J]. Nat. Gas Industry 18 (04).
Ozkan, E., Ohaeri, U., and Raghavan, R. (1987). Unsteady Flow to a Well-Produced at a Constant Pressure in a Fractured Reservoir [J]. SPE Formation Eval. 2 (2), 186-200. doi:10.2118/9902-pa

Sun, E. H., Li, X. P., and Wang, W. D. (2012). Study on Productivity Analysis Method of Gas-Water Two-phase Flow Well in Low Permeability Gas Reservoir [J]. Lithologic Reservoir 12 (06). doi:10.3969/j.issn.16738926.2012.06.025

Wang, S., Zhang, S., Tan., X., Jiang, H., and Zhang, X. (2017). Numerical Simulation Method and Laboratory Experiments of Starting Pressure Gradient in Tight Sandstone Oil Reservoirs. SPE/IATMI Asia pacific Oil Gas Conference and Exhibition. doi:10.2118/186349-MS

Warren, J. E., and Root, P. J. (1963). The Behavior of Naturally Fractured Reservoirs [J]. Soc. Pet. Eng. J. 3 (3), 245-255. doi:10.2118/426-pa

Conflict of Interest: The authors declare that the research was conducted in the absence of any commercial or financial relationships that could be construed as a potential conflict of interest.

Publisher's Note: All claims expressed in this article are solely those of the authors and do not necessarily represent those of their affiliated organizations, or those of the publisher, the editors, and the reviewers. Any product that may be evaluated in this article, or claim that may be made by its manufacturer, is not guaranteed or endorsed by the publisher.

Copyright (c) 2021 Wang, Tan, Xia, Tian and Liang. This is an open-access article distributed under the terms of the Creative Commons Attribution License (CC BY). The use, distribution or reproduction in other forums is permitted, provided the original author(s) and the copyright owner(s) are credited and that the original publication in this journal is cited, in accordance with accepted academic practice. No use, distribution or reproduction is permitted which does not comply with these terms. 